\title{
Associação entre o estado nutricional e a ingestão dietética em pacientes com fibrose cística*
}

\author{
Association between nutritional status and \\ dietary intake in patients with cystic fibrosis \\ Míriam Isabel Souza dos Santos Simon, Michele Drehmer, \\ Sérgio Saldanha Menna-Barreto
}

\begin{abstract}
Resumo
Objetivo: Determinar a relação entre o estado nutricional e a ingestão dietética de pacientes com fibrose cística. Métodos: Estudo transversal com 85 pacientes com fibrose cística entre 6 e 18 anos de idade. A ingestão dietética foi avaliada pelo registro alimentar de 3 dias com a pesagem dos alimentos consumidos. Os desfechos avaliados foram os seguintes indicadores do estado nutricional: percentual da relação peso/estatura (\%P/E), percentil do índice de massa corpórea (IMC), escore Z para estatura/idade (E/1) e peso/idade ( $\mathrm{P} / 1)$, e percentual de ingestão dietética comparada a Recommended Dietary Allowances (RDAs). Resultados: A prevalência de pacientes eutróficos foi de 77,7\%, considerando o IMC acima do percentil 25 como ponto de corte, e 83,5\% estavam acima de 90\% do \%P/E. A média de ingestão, avaliada em 82 pacientes, foi de 124,5\% da RDA. Nas análises de regressão logística univariada, encontrou-se uma associação significativa entre a variável independente ingestão calórica e o desfecho escore Z E/1. 0 modelo de análise multivariado, elaborado a partir do desfecho escore Z E/1 e ajustado para idade, $\mathrm{VEF}_{1}$, colonização por Staphylococcus aureus resistente à meticilina e número de internações hospitalares, demonstrou que um aumento de 1\% da ingestão calórica em relação à RDA diminui em 2\% a chance de ter déficit de estatura (OR =0,98; 1C95\%: 0,96-1,00). A escolaridade materna demonstrou uma associação limítrofe ( $p$ = 0,054). Conclusões: Houve baixa prevalência de desnutrição nesta amostra. 0 modelo de estudo demonstrou evidências da associação entre a ingestão dietética e o estado nutricional, sendo esta ingestão um fator preditor de crescimento nesses pacientes.
\end{abstract}

Descritores: Fibrose cística; Estado nutricional; Registros de dieta; Criança; Adolescente.

\begin{abstract}
Objective: To determine the relationship between nutritional status and dietary intake in patients with cystic fibrosis. Methods: Cross-sectional study involving 85 cystic fibrosis patients between 6 and 18 years of age. Dietary intake was evaluated by the 3-day diet record (weighing the food consumed). The outcome measures were the following nutritional status indicators: weight/height $(\mathrm{W} / \mathrm{H} \%)$ percentage, body mass index (BMI) percentiles, $\mathrm{Z}$ score for weight/age $(\mathrm{W} / \mathrm{A}), \mathrm{Z}$ score for height/age $(\mathrm{H} / \mathrm{A})$ and percentage of dietary intake compared with the Recommended Dietary Allowance (RDA). Results: The prevalence of well-nourished patients was 77.7\%, using BMl above the 25th percentile as the cut-off value, and the $\mathrm{W} / \mathrm{H} \%$ was above $90 \%$ in $83.5 \%$. The mean dietary intake, evaluated in 82 patients, was $124.5 \%$ of the RDA. In the univariate logistic regression analyses, we found a significant association between the independent variable calorie intake and the $\mathrm{Z}$ score for W/A. The multivariate analysis, based on the $\mathrm{Z}$ score for H/A and adjusted for $\mathrm{FEV}_{1}$, methicillin-resistant Staphylococcus aureus colonization and number of hospitalizations, demonstrated that a $1 \%$ increase in the calorie intake decreases the chance of having short stature by 2\% (OR: 0.98; 95\% Cl: 0.96-1.00). Maternal level of education showed a borderline association ( $p=0.054)$. Conclusions: The prevalence of malnutrition was low in this sample of patients. The study model demonstrated an association between dietary intake and nutritional status. Dietary intake was a predictive factor of statural growth in patients with cystic fibrosis.
\end{abstract}

Keywords: Cystic fibrosis; Nutritional status; Diet records; Child; Adolescent.

\footnotetext{
* Trabalho realizado no Hospital de Clínicas de Porto Alegre, Faculdade de Medicina, Universidade Federal do Rio Grande do Sul UFRGS - Porto Alegre (RS) Brasil.

Endereço para correspondência: Míriam lsabel Souza dos Santos Simon. Av. Ramiro Barcelos, 2350, CEP 90035-903, Porto Alegre, RS, Brasil.

Tel 5551 2101-8410.E-mail: misantos@hcpa.ufrgs.br

Apoio financeiro: Este estudo recebeu apoio financeiro do Fundo de Incentivo à Pesquisa (FIPE) e do Grupo de Pós-Graduação e Pesquisa (GPPG) do Hospital de Clínicas de Porto Alegre.

Recebido para publicação em 3/9/2008. Aprovado, após revisão, em 1/6/2009.
} 


\section{Introdução}

A desnutrição é uma importante causa de mortalidade em crianças, jovens e adultos com fibrose cística (FC), estando relacionada à progressão dessa doença. Os fatores que contribuem para a perpetuação do agravamento nutricional podem estar relacionados ao aumento da necessidade nutricional, à redução da ingestão e ao aumento das perdas. ${ }^{(1)}$

0 aumento da necessidade energética, a diminuição da ingestão e o aumento das perdas estão relacionados com a deterioração da função pulmonar, anorexia, vômitos, insuficiência pancreática, atividade inflamatória crônica e complicações biliares e intestinais, levando à consequente perda de massa magra e depleção da função imunológica. ${ }^{(2)}$

A recomendação de ingestão diária de energia baseia-se na elevação do gasto energético apresentado por esses pacientes. Admite-se que os pacientes portadores de FC necessitam de, pelo menos, $120-150 \%$ da energia estabelecida pelas Recommended Dietary Allowances (RDAs) de 1989.. ${ }^{(3)}$ Nada indica precisamente qual a porcentagem dessa maior necessidade, pois ela é dependente da má absorção intestinal e da aceleração do metabolismo. ${ }^{(2)}$

Apesar dos avanços do tratamento clínico e nutricional, a Cystic Fibrosis Foundation, que compila os dados dos pacientes americanos com FC, relatou que 15,7\% e 16,3\% desses pacientes apresentavam valores abaixo do percentil 5 para peso e estatura, respectivamente, em 2004. ${ }^{(4)} \mathrm{Em}$ 1993, o Registro Latino Americano de Fibrose Cística demonstrou que mais de 50\% dos pacientes se encontravam abaixo do percentil 3 de peso e que $46,7 \%$ dos pacientes estavam abaixo do mesmo percentil na relação estatura para idade. ${ }^{(5)}$

0 padrão de ingestão dietética em pacientes com FC parece ter uma relação direta com o crescimento e o estado nutricional dos mesmos; por outro lado, a estatura também pode estar relacionada à sobrevida nesses pacientes. ${ }^{(6)}$ Este estudo objetivou determinar a relação entre o estado nutricional e a ingestão dietética de pacientes com FC de 6-18 anos de idade atendidos em um centro de referência no Brasil.

\section{Métodos}

Trata-se de um estudo transversal cujos dados foram coletados entre julho de 2004 e dezembro de 2005, durante o acompanhamento ambulatorial de rotina dos pacientes com FC. Foram incluídos, consecutivamente, todos os pacientes $(\mathrm{n}=88)$ com FC, com idades entre 6 e 18 anos, não colonizados por Burkholderia cepacia e em acompanhamento pela equipe de Pneumologia Pediátrica do Hospital de Clínicas de Porto Alegre (HCPA), RS. Para sua inclusão no estudo, os pacientes deveriam ter história clínica de FC e dosagem de sódio e cloreto elevados no suor ou a identificação de duas mutações. Foram excluídos 2 pacientes com doença terminal e 1 paciente devido à impossibilidade de se obter o termo de consentimento livre e esclarecido.

Foi utilizado como instrumento para a coleta de dados uma ficha onde constavam os seguintes campos: data de nascimento, gênero, idade, escolaridade da mãe do paciente, suplementação enzimática e vitamínica, colonização bacteriana, $\mathrm{VEF}_{1}$ e número de internações hospitalares desde o diagnóstico. A insuficiência pancreática foi determinada pela avaliação do uso de enzimas pancreáticas. A colonização bacteriana foi baseada nas amostras de escarro dos últimos doze meses.

A espirometria foi realizada para a aferição de $\mathrm{VEF}_{1}$, parâmetro mais comumente empregado para quantificar o defeito ventilatório obstrutivo, característico na FC. Foram realizadas curvas fluxo-volume através de um espirômetro Master Screen (Jaeger, Würzburg, Alemanha), utilizando-se a tabela de Zapletal et al. para os valores previstos. ${ }^{(7)} \mathrm{A}$ espirometria foi realizada sempre pelo mesmo avaliador, e sua qualidade foi avaliada pelo médico assistente mediante a análise das curvas.

A ingestão dietética foi avaliada pelo registro alimentar de três dias, não consecutivos, com pesagem dos alimentos consumidos. Para a pesagem dos alimentos foi fornecida uma balança com capacidade máxima de $2 \mathrm{~kg}$ e com divisão mínima de $25 \mathrm{~g}$; para medir os líquidos, foi fornecido um copo medidor de plástico tipo béquer com capacidade para $600 \mathrm{~mL}$ e com divisões mínimas de $10 \mathrm{~mL}$. 0 paciente e/ou os pais (ou responsáveis) foram treinados para preencher corretamente esse registro alimentar de três dias. ${ }^{(8)}$ Esse inquérito foi realizado referente a dois dias da semana não consecutivos e um dia de fim de semana, sendo que todas as preparações caseiras deveriam ser anotadas, com os devidos ingredientes utilizados, além da quan- 
tidade de óleo utilizada por mês pela família. 0 cálculo do registro alimentar foi realizado através do programa Sistema de Apoio à Decisão em Nutrição da Escola Paulista de Medicina ${ }^{(9)}$ e comparado com as RDAs. ${ }^{(3)}$

A avaliação antropométrica ocorreu durante a consulta, após a obtenção do termo de consentimento livre e esclarecido pelos responsáveis. 0 peso e a estatura foram mensurados sempre pelo mesmo avaliador através de técnicas padronizadas. 0 peso foi obtido, com o paciente vestindo apenas um avental padronizado, em uma balança eletrônica (Filizola, São Paulo, Brasil) com carga máxima de $150 \mathrm{~kg}$ e variação de $50 \mathrm{~g}$. A estatura foi mensurada em um estadiômetro fixo na parede da marca Sanny (American Medical do Brasil, São Bernardo do Campo, Brasil) com o paciente sem calçados ou adereços no cabelo, com os calcanhares unidos, de costas para o antropômetro, os braços relaxados ao longo do corpo e a cabeça na posição vertical, com os olhos fixos à frente. Enquanto o avaliado inspirava, o cursor horizontal era abaixado até o ponto mais alto da cabeça. A medida da circunferência do braço (CB) foi obtida no ponto médio do braço não dominante com uma fita métrica flexível e inextensível. A dobra cutânea tricipital (DCT) foi medida no ponto médio do braço não dominante com um compasso de dobras cutâneas (Harpenden; British Indicators, Burguess Hill, Reino Unido). A circunferência muscular do braço (CMB) foi calculada pela seguinte equação: CMB $(\mathrm{cm})=$ CB - DCT $(\mathrm{mm}) \times 0,314$. Todos os valores foram comparados com os critérios estabelecidos por Frisancho. ${ }^{(10)}$

Os parâmetros nutricionais utilizados foram o escore $\mathrm{Z}$ para os índices peso/idade $(\mathrm{P} / \mathrm{I})$ e estatura/idade (E/I), conforme padrões do National Center for Health Statistics, percentil de índice de massa corpórea (PIMC) e a relação peso/estatura em porcentagem $(\% \mathrm{P} / \mathrm{E})$, calculada através da divisão entre o peso atual e o peso do percentil correspondente à estatura $\times$ 100. Esses dois últimos parâmetros foram utilizados por serem os indicadores nutricionais recomendados em dois consensos, ${ }^{(11,12)}$ nos quais foram estabelecidos, como pontos de corte para determinar falha nutricional, risco nutricional e desnutrição, respectivamente, valores abaixo de $90 \%$ de $\%$ P/E, PIMC entre os percentis 10 e 25 , e PIMC abaixo do percentil 10.
Para a análise dos dados, foram calculadas frequências, médias e medianas das variáveis dependentes e independentes. Foi utilizada também a regressão logística univariada entre os fatores em estudo com os desfechos que representavam o estado nutricional $(\% \mathrm{P} / \mathrm{E}$, escore $\mathrm{Z}$ $\mathrm{E} / 1$, escore $\mathrm{Z} \mathrm{P} / 1$ e PIMC). Foram incluídas no modelo multivariado as variáveis que tiveram $p \leq 0,25$. 0 nível de significância adotado foi de 0,05 , e as análises foram realizadas através do programa Statistical Package for the Social Sciences, versão 12.0 (SPSS Inc., Chicago, IL, EUA).

0 estudo foi aprovado pela Comissão de Pesquisa e Ética em Saúde do Grupo de Pesquisa e Pós-Graduação do HCPA (Projeto 03-389). Os recursos necessários para a compra de balanças e copos medidores foram solicitados ao Fundo de Incentivo a Pesquisa.

\section{Resultados}

A amostra consistiu de 85 pacientes, sendo 55,3\% do sexo masculino, com média de idade de 11,2 $\pm 3,2$ anos. A idade variou entre 6,0 e 17,5 anos. Desses pacientes, 39 (45,9\%) eram

Tabela 1 - Indicadores do estado nutricional e do perfil dietético de pacientes com fibrose cística atendidos em um ambulatório especializado no sul do Brasil.

\begin{tabular}{lc}
\hline \multicolumn{1}{c}{$\begin{array}{c}\text { Indicadores do estado nutricional } \\
(\mathrm{n}=85)\end{array}$} & Valores \\
\hline IMC, percentil & $45,1 \pm 26,5$ \\
Peso/estatura, \% & $100,4 \pm 13,0$ \\
Escore Z peso/idade & $-0,27 \pm 1,2$ \\
Escore Z estatura/idade & $-0,24 \pm 1,1$ \\
Avaliação da composição corporal ${ }^{a}$ & \\
$\quad$ DCT < Percentil 5 & $9(10,6)$ \\
DCT > Percentil 5 & $76(89,4)$ \\
CMB < Percentil 5 & $14(16,5)$ \\
CMB > Percentil 5 & $71(83,5)$ \\
$\quad$ Total & $85(100,0)$ \\
Perfil dietético (n = 82) & \\
$\quad$ Calorias em relação à RDA, \% & $124,5 \pm 34,5$ \\
$\quad$ Carboidratos, \% & $55,3 \pm 8,7$ \\
$\quad$ Proteínas, \% & $14,6 \pm 3,9$ \\
$\quad$ Lipídios, \% & $29,9 \pm 6,8$
\end{tabular}

IMC: índice de massa corpórea; DCT: dobra cutânea tricipital; CMB: circunferência muscular do braço; e RDA: Recommended Dietary Allowance. Dados apresentados em média $\pm \mathrm{dp}$ ou n (\%). ${ }^{\mathrm{a} C o m p o s i c ̧ a ̃ o ~ c o r p o r a l ~ a v a l i a d a ~}$ segundo critérios de Frisancho. ${ }^{(10)}$ 


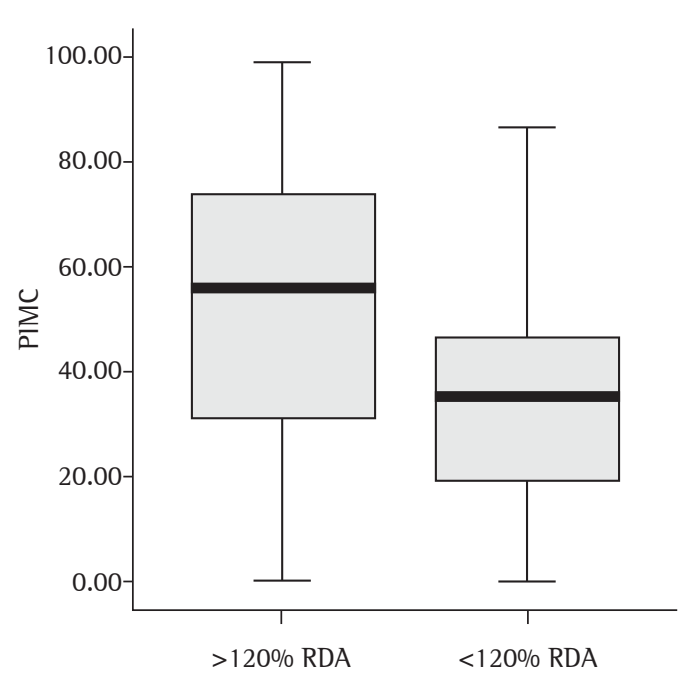

Figura 1 - Disposição gráfica do tipo box plot da distribuição da amostra: medianas do percentil do índice de massa corpórea (PIMC) em relação ao consumo calórico acima ou abaixo do recomendado, em relação à Recommended Dietary Allowance (RDA) para pacientes com fibrose cística (120\% da RDA).

procedentes de Porto Alegre e de sua região metropolitana, $17(20,0 \%)$ eram da região nordeste do estado, e os restantes pertenciam às demais regiões do estado. A insuficiência pancreática estava presente em $87,1 \%$ dos pacientes. A suplementação vitamínica era utilizada regularmente por $97,6 \%$ dos pacientes. Os suplementos nutricionais regularmente utilizados por esses pacientes eram polímeros de glicose e dieta líquida hipercalórica com 1,5 kcal/mL; 65,9\% dos pacientes utilizavam um ou mais suplementos v.o., e 9,4\% faziam suplementação noturna por gastrostomia.

A média de anos de escolaridade materna foi de 9,0 $\pm 3,8$ anos, e 34\% delas apresen-

Tabela 2 - Regressões logísticas univariadas entre os indicadores do estado nutricional e o percentual de ingestão calórica ajustado à Recommended Dietary Allowance em pacientes com fibrose cística.

\begin{tabular}{lcc}
\hline $\begin{array}{c}\text { Ingestão calórica } \\
\text { ajustada à RDA }\end{array}$ & OR (1C95\%) & $p$ \\
\hline Peso/estatura, \% & $0,99(0,98-1,01)$ & 0,66 \\
Escore Z estatura/idade & $0,97(0,96-0,99)$ & 0,019 \\
Escore Z peso/idade & $0,99(0,97-1,00)$ & 0,31 \\
IMC, percentil & $0,99(0,98-1,01)$ & 0,37 \\
\hline IMC: índice de massa corpórea; e RDA: Recommended \\
Dietary Allowance.
\end{tabular}

tavam o ensino fundamental incompleto. Quanto à presença de colonização bacteriana no último ano, 74,1\% dos pacientes apresentaram Staphylococcus aureus, 18,8\% apresentaram $S$. aureus resistente à meticilina (MRSA, do inglês methicillin-resistant S. aureus), 52,9\% apresentaram Pseudomonas aeruginosa, e 24,7\% apresentavam $P$. aeruginosa mucoide. A média de $\mathrm{VEF}_{1}$ foi de $84,1 \pm 24,4 \%$. A mediana do número de internações foi igual a 5 , com intervalo interquartil entre 2 e 11 .

De acordo com os quatro indicadores do estado nutricional avaliados neste estudo, todas as médias ficaram dentro dos pontos de corte para eutrofia, demonstrando o adequado estado nutricional encontrado nesta amostra (Tabela 1). Neste estudo, 16,5\% dos pacientes apresentavam $\% \mathrm{P} / \mathrm{E}$ igual ou abaixo de 90\%, 14,1\% tinham PIMC entre os percentis 10 e 25 , e $8,2 \%$ tinham PIMC abaixo do percentil 10.

Em relação à composição corporal, foi encontrada uma depleção de reserva de gordura, pela medição da DCT, em 10,6\% dos pacientes. Houve depleção de massa muscular, estimada pela CMB, em 16,5\% dos pacientes (Tabela 1).

A média percentual de ingestão calórica, avaliada em 82 pacientes, foi de $124,6 \%$ da RDA. A prevalência de pacientes que apresentaram ingestão energética acima de 120\% da RDA foi de 51,7\% (Tabela 1).

Na Figura 1, demonstra-se que os pacientes que ingeriam calorias acima de $120 \%$ da RDA tinham mediana de PIMC com valor de 56; já os que ingeriam calorias abaixo dessas recomendações, mantinham uma mediana de PIMC igual a 34,9 .

A Tabela 2 apresenta a associação entre a ingestão calórica e o estado nutricional, avaliado pelas variáveis dependentes $\% \mathrm{P} / \mathrm{E}, \mathrm{PIMC}$, escore $\mathrm{Z}$ E/l e escore Z P/l mediante análises de regressão logística univariadas. Os indicadores do estado nutricional avaliados foram dicotomizados, utilizando-se os pontos de corte para desnutrição conforme consensos de $\mathrm{FC}^{(11,12)}$ e da Organização Mundial da Saúde. ${ }^{(13)} \mathrm{Na}$ análise bruta, houve uma associação significativa entre a variável independente ingestão calórica e o desfecho escore Z E/l.

0 modelo de análise multivariado foi elaborado a partir do desfecho escore $\mathrm{Z} E / \mathrm{l}$, já que a associação desse desfecho com a ingestão calórica foi verificada na análise bruta. Baseando-se 
Tabela 3 - Modelo final de regressão logística multivariada, relacionando o desfecho escore Z de estatura/idade com ingestão calórica e escolaridade materna.

\begin{tabular}{lcccc}
\hline \multicolumn{1}{c}{ Variáveis } & Beta (erro-padrão) & Teste de Wald & $p$ & OR (1C95\%) \\
\hline Ingestão calórica ajustada à RDA & $-0,019(0,010)$ & 3,96 & 0,046 & $0,98(0,96-1,00)$ \\
Escolaridade materna & $-0,16(0,08)$ & 3,72 & 0,054 & $0,85(0,72-1,00)$ \\
\hline
\end{tabular}

RDA: Recommended Dietary Allowances. Modelo final ajustado para idade, $\mathrm{VEF}_{1}$, colonização por S. aureus resistente à meticilina e número de internações hospitalares.

na hipótese de que a ingestão calórica está associada ao crescimento, foram incluídas no modelo multivariado as variáveis independentes que tiveram, na análise bruta com o escore $\mathrm{Z}$ $E / 1$, um valor $p$ de até 0,25 . Assim, utilizando-se o escore Z E/l, dicotomizado em eutrofia e não-eutrofia (acima de -1,28), foram incluídas no modelo as seguintes variáveis: ingestão calórica ajustada à RDA, escolaridade da mãe, colonização por MRSA, $\mathrm{VEF}_{1}$, idade e número de internações hospitalares. Esse modelo de regressão logística, conforme o teste estatístico de Hosmer e Lemeshow, mostrou-se ajustado (qui-quadrado $=5,57 ; p=0,7$ ).

0 aumento de um percentual, em relação à RDA, de ingestão calórica, diminui em $2 \%$ a chance de haver déficit de estatura $(O R=0,98$; 1C95\%: 0,96-1,00), ajustando-se para idade, $\mathrm{VEF}_{1}$, colonização por MRSA e número de internações hospitalares (Tabela 3). A escolaridade materna parece influenciar o indicador nutricional relacionado com o crescimento, ou seja, parece haver um efeito protetor da maior escolaridade da mãe sobre o indicador nutricional relacionado ao crescimento. Entretanto, na análise multivariada, essa associação permaneceu limítrofe para a significância estatística $(p=0,054)$.

\section{Discussão}

Os parâmetros nutricionais desta amostra podem ser comparados com os de pacientes atendidos nos centros de FC de países desenvolvidos ${ }^{(4)}$ devido à baixa prevalência de desnutrição, mesmo se avaliados através dos critérios rigorosos estabelecidos pelos consensos de FC e considerando-se as condições socioeconômicas de um país em desenvolvimento. Supõe-se que tais achados possam estar relacionados ao manejo interdisciplinar de um centro de referência, como observado em um estudo. ${ }^{(14)} \mathrm{Um}$ estudo norte-americano com 22.714 pacientes com FC demonstrou uma tendência à melhora do estado nutricional por idade nesses pacientes na última década. ${ }^{(4)}$

Dados do United Kingdom Cystic Fibrosis Registry revelaram que a média do escore $\mathrm{Z}$ do peso de meninos de até 10 anos de idade foi de $-0,25$ à $-0,5$; após essa idade, houve declínio no IMC. As meninas apresentavam um escore $\mathrm{Z}$ de $-0,5$; tendo uma queda no IMC após 5 anos de seguimento. ${ }^{(15)}$ No presente estudo, dados semelhantes foram encontrados como, por exemplo, o escore $\mathrm{Z} \mathrm{P} / 1$ e o escore $\mathrm{Z}$ E/1 tiveram médias iguais a $-0,27 \pm 1,16$ e $-0,24 \pm 1,07$, respectivamente.

No presente estudo, a depleção de reserva de gordura e a e depleção de massa muscular foram encontradas em 10,6\% e 16,5\% dos pacientes, respectivamente. Em um estudo na cidade de Florianópolis com 13 pacientes com idades entre 2 e 20 anos, $46,1 \%$ dos pacientes apresentaram depleção muscular. ${ }^{(16)} \mathrm{A}$ análise da composição corporal em pacientes com FC é justificada devido ao estado crônico de estresse catabólico ou de desnutrição ligados a exacerbações pulmonares que afetam adversamente o balanço energético e o metabolismo proteico, comprometendo principalmente a reserva corporal proteica.

A boa aderência ao tratamento nutricional nesta amostra também pode ser observada pela ingestão energética. A média de ingestão, comparada com a RDA, dos 82 pacientes avaliados foi de $124,56 \%$ da RDA, sendo a distribuição média de nutrientes da seguinte forma: $14,6 \%$ de proteínas, 29,9\% de lipídios e 55,2\% de carboidratos. Um estudo conduzido com adolescentes revelou que meninos e meninas com crescimento normal consumiam em média 110\% da RDA. ${ }^{(17)}$ Outro estudo demonstrou que a ingestão de nutrientes em pacientes com FC era semelhante à recomendação para idade e sexo. ${ }^{(18)}$ Alguns autores encontraram uma média de ingestão energética de $117 \%$ da RDA e percentuais de nutrientes de $35,7 \%$ de lipídios, $47,9 \%$ de carboidratos e $15,2 \%$ de proteínas, sendo que somente $40 \%$ dos pacientes atingiram a recomendação de 
$120 \%$ da RDA. ${ }^{(19)}$ Os percentuais de macronutrientes encontrados no presente estudo estão dentro do padrão recomendado para a população em geral, evidenciando um equilíbrio na distribuição da dieta. No entanto, a contribuição dos alimentos ricos em gordura poderia ser um pouco maior em detrimento dos carboidratos.

0 presente estudo, por tratar-se de uma análise transversal, aponta apenas para as associações, sem, contudo, ser possivel estabelecer uma relação de causa e efeito entre a ingestão alimentar e o comprometimento do estado nutricional.

A ingestão calórica foi associada somente ao indicador nutricional escore Z E/l nas análises brutas. 0 déficit de estatura para a idade pode representar a presença de desnutrição intrauterina ou a presença crônica de desnutrição. ${ }^{(20)}$ Há evidências de que redução de altura é mais importante para determinar a sobrevida que a redução de peso para estatura na $F C .{ }^{(6,21)} 0$ efeito da baixa estatura na sobrevida pode ser influenciado por falha de crescimento em um período da vida de importante crescimento e desenvolvimento pulmonar. ${ }^{(6)}$

$\mathrm{Na}$ análise multivariada, ajustando-se para colonização bacteriana por MRSA, idade, número de internações e $\mathrm{VEF}_{1}$, a baixa escolaridade materna apresentou uma associação limítrofe para o déficit estatural. Em um estudo sobre a relação da capacidade materna de cuidar e a desnutrição, observou-se que a baixa escolaridade materna duplicou o risco de desnutrição. ${ }^{(22)}$ Alguns autores verificaram que pacientes com FC mais pobres apresentavam valores de função pulmonar e estado nutricional mais deteriorados, além de um risco de morrer 3,65 vezes maior. ${ }^{(23)}$ Outro estudo recente demonstrou que pacientes com melhor nível socioeconômico apresentam risco 40\% menor em relação à mortalidade quando comparados com pacientes de nível socioeconômico inferior. ${ }^{(24)}$

Por outro lado, a ingestão calórica no modelo multivariado foi um fator preditor de estatura em pacientes com FC, ou seja, quanto maior a ingestão calórica, menor o risco de desnutrição por déficit estatural, ajustando-se para idade, colonização por MRSA, VEF , número de internações hospitalares e anos de escolaridade da mãe. Um estudo demonstrou um aumento significativo no escore $\mathrm{Z}$ E/l após uma intervenção nutricional, através da qual os pacientes com
FC atingiram uma ingestão calórica de 132\% da RDA. ${ }^{(25)}$

A importância do estado nutricional em pacientes com FC é bem conhecida e tem sido largamente documentada. 0 presente trabalho demonstra evidências da associação da ingestão calórica com o estado nutricional, relacionando o perfil dietético como um fator preditor de estatura nesses pacientes.

\section{Agradecimentos}

Aos pacientes que se dispuseram a colaborar com o estudo. Ao HCPA - Serviço de Pneumologia, Seção de Pneumologia Pediátrica e Serviço de Nutrição e Dietética, agradecemos o apoio.

\section{Referências}

1. Dodge JA, Turck D. Cystic fibrosis: nutritional consequences and management. Best Pract Res Clin Gastroenterol. 2006;20(3):531-46.

2. Koletzko S, Reinhardt D. Nutritional challenges of infants with cystic fibrosis. Early Hum Dev. 2001;65 Suppl:S53-61.

3. National Research Council (U.S.). Subcommittee on the Tenth Edition of the RDAs.; National Institutes of Health (U.S.); National Research Council (U.S.). Committee on Dietary Allowances. Recommended dietary allowances. Washington: National Academy Press; 1989.

4. Cystic Fibrosis Foundation. Patient Registry 2004 Annual date report. Bethesda: Cystic Fibrosis Foundation; 2005.

5. Registro Latino-Americano de Fibrosis Quística (REGLAFQ). Informe del cuarto año. Buenos Aires; 1993. p.21.

6. Beker LT, Russek-Cohen E, Fink RJ. Stature as a prognostic factor in cystic fibrosis survival. J Am Diet Assoc. 2001;101(4):438-42.

7. Zapletal A, Motoyama EK, Gibson LE, Bouhuys A. Pulmonary mechanics in asthma and cystic fibrosis. Pediatrics. 1971;48(1):64-72.

8. Moulin CC, Tiskievicz F, Zelmanovitz T, de Oliveira J, Azevedo MJ, Gross JL. Use of weighed diet records in the evaluation of diets with different protein contents in patients with type 2 diabetes. Am J Clin Nutr. 1998;67(5):853-7.

9. Anção MS, Cuppari L, Tudisco LS, Draibe AS, Sigulem D. Sistema de apoio à decisão em nutrição-versão 2.5. São Paulo: Centro de Informática em Saúde da Universidade Federal de São Paulo/Escola Paulista de Medicina; 1993.

10. Frisancho AR. New norms of upper limb fat and muscle areas for assessment of nutritional status. Am J Clin Nutr. 1981;34(11):2540-5.

11. Sinaasappel M, Stern M, Littlewood J, Wolfe S, Steinkamp G, Heijerman HG, et al. Nutrition in patients with cystic fibrosis: a European Consensus. J Cyst Fibros. 2002;1(2):51-75. 
12. Borowitz D, Baker RD, Stallings V. Consensus report on nutrition for pediatric patients with cystic fibrosis. J Pediatr Gastroenterol Nutr. 2002;35(3):246-59.

13. WHO Expert Committee on Physical Status. Physical Status: The Use and Interpretation of Anthropometry. Report of a WHO Expert Committee. Geneva: The Organization; 1995. WHO Technical Report Series No.: 854.

14. Collins CE, MacDonald-Wicks L, Rowe S, O’Loughlin EV, Henry RL. Normal growth in cystic fibrosis associated with a specialised centre. Arch Dis Child. 1999;81(3):241-6.

15. Morison S, Dodge JA, Cole TJ, Lewis PA, Coles EC, Geddes $D$, et al. Height and weight in cystic fibrosis: a cross sectional study. UK Cystic Fibrosis Survey Management Committee. Arch Dis Child. 1997;77(6):497-500.

16. Fiates GM, Barbosa E, Auler F, Feiten SF, Miranda F. Estado nutricional e ingestão alimentar de pessoas com fibrose cística. Rev Nutri. 2001;14(2):95-101.

17. Bentur L, Kalnins D, Levison H, Corey M, Durie PR. Dietary intakes of young children with cystic fibrosis: is there a difference? J Pediatr Gastroenterol Nutr. 1996;22(3):254-8.
18. Powers SW, Patton SR, Byars KC, Mitchell MJ, Jelalian E, Mulvihill MM, et al. Caloric intake and eating behavior in infants and toddlers with cystic fibrosis. Pediatrics. 2002;109(5):E75-5.

19. White H, Morton AM, Peckham DG, Conway SP. Dietary intakes in adult patients with cystic fibrosis--do they achieve guidelines? J Cyst Fibros. 2004;3(1):1-7.

20. Vitolo MR. Avaliação nutricional da criança. In: Vitolo MR, editor. Nutrição, da gestação à adolescência. Rio de Janeiro: Reichmann \& Affonso; 2003. p. 99.

21. Hayllar KM, Williams SG, Wise AE, Pouria S, Lombard M, Hodson ME, et al. A prognostic model for the prediction of survival in cystic fibrosis. Thorax. 1997;52(4):313-7.

22. Carvalhaes MA, Benício MH. Mother's ability of childcare and children malnutrition [Article in Portuguese]. Rev Saude Publica. 2002;36(2):188-97.

23. Schechter MS, Shelton BJ, Margolis PA, Fitzsimmons SC. The association of socioeconomic status with outcomes in cystic fibrosis patients in the United States. Am J Respir Crit Care Med. 2001;163(6):1331-7.

24. O'Connor GT, Quinton HB, Kahn R, Robichaud P, Maddock J, Lever T, et al. Case-mix adjustment for evaluation of mortality in cystic fibrosis. Pediatr Pulmonol. 2002;33(2):99-105.

25. Gaspar MC, Chiba SM, Gomes CE, Juliano Y, Novo NF, Ancona-Lopez F. Resultado de intervenção nutricional em crianças e adolescentes com fibrose cística. J Pediatr (Rio J). 2002;78(2):161-70.

\section{Sobre os autores}

\section{Míriam Isabel Souza dos Santos Simon}

Chefe do Serviço de Nutrição e Dietética. Hospital de Clínicas de Porto Alegre, Faculdade de Medicina, Universidade Federal do Rio Grande do Sul - UFRGS - Porto Alegre (RS) Brasil.

\section{Michele Drehmer}

Doutorando em Epidemiologia. Universidade Federal do Rio Grande do Sul - UFRGS - Porto Alegre (RS) Brasil.

\section{Sérgio Saldanha Menna-Barreto}

Professor Titular. Departamento de Medicina Interna, Faculdade de Medicina, Universidade Federal do Rio Grande do Sul - UFRGS Porto Alegre (RS) Brasil. 